This document was prepared in conjunction with work accomplished under Contract No. DE-AC09-96SR18500 with the U.S. Department of Energy.

This work was prepared under an agreement with and funded by the U.S. Government. Neither the U. S. Government or its employees, nor any of its contractors, subcontractors or their employees, makes any express or implied: 1 . warranty or assumes any legal liability for the accuracy, completeness, or for the use or results of such use of any information, product, or process disclosed; or 2 . representation that such use or results of such use would not infringe privately owned rights; or 3 . endorsement or recommendation of any specifically identified commercial product, process, or service. Any views and opinions of authors expressed in this work do not necessarily state or reflect those of the United States Government, or its contractors, or subcontractors. 


\title{
Derivations for Hoop Stresses Due to Shock Waves in a Tube
}

\author{
Robert A. Leishear \\ Washington Savannah River Company \\ Aiken, South Carolina, 29803 \\ 803-725-2832, Robert.Leishear@SRS.gov
}

\begin{abstract}
Equations describing the hoop stresses in a pipe due to water hammer have been presented in the literature in a series of papers, and this paper discusses the complete derivation of the pertinent equations. The derivation considers the pipe wall response to a water hammer induced shock wave moving along the inner wall of the pipe. Factors such as fluid properties, pipe wall materials, pipe dimensions, and damping are considered. These factors are combined to present a single, albeit rather complicated, equation to describe the pipe wall vibrations and hoop stresses as a function of time. This equation is also compared to another theoretical prediction for hoop stresses, which is also derived herein. Specifically, the two theories predict different maximum stresses, and the differences between these predictions are graphically displayed.
\end{abstract}

\section{KEYWORDS}

Water hammer, hoop stress, dynamic stress, breathing stress.

\section{SYMBOLS}

$\begin{array}{ll}\text { A } & \text { area, inch }{ }^{2} \\ \mathrm{~A}_{1}-\mathrm{A}_{8} & \text { arbitrary constants } \\ \mathrm{a}, \mathrm{b}, \mathrm{c}, \mathrm{d}, \mathrm{s} & \text { constants } \\ \mathrm{c} & \text { circumference, inches } \\ \mathrm{C} & \text { damping } \\ \mathrm{D} & \text { flexural rigidity } \\ \mathrm{Ds} & \text { specific damping } \\ \mathrm{DLF} & \text { dynamic load factor } \\ \mathrm{E} & \text { elastic modulus, psi } \\ \mathrm{F} & \text { force, pound force } \\ \mathrm{H} & \text { Heaviside step function } \\ \mathrm{Hz} & \text { Hertz, cycle per second } \\ \mathrm{ID} & \text { inside diameter, inches } \\ \mathrm{i} & \text { impact factor } \\ \mathrm{g} & \text { gravitational constant } \\ \mathrm{K} & \text { spring constant } \\ \mathrm{k} & \text { bulk modulus, psi } \\ \mathrm{L} & \text { length, inch } \\ \mathrm{N}_{\mathrm{x}} & \text { force per unit length, pound / inch } \\ \mathrm{N}_{\theta} & \text { hoop stress resultant, pound / inch } \\ \mathrm{NPS} & \text { nominal pipe size } \\ \mathrm{M} & \text { moment, inch -pound }\end{array}$

\begin{tabular}{|c|c|}
\hline $\mathrm{m}$ & mass, pound mass \\
\hline OD & outside diameter \\
\hline $\mathrm{P}, \mathrm{P}_{0}$ & radial load, psi \\
\hline P.O. & percent overshoot \\
\hline psi & pounds per square inch \\
\hline Q & shear force / unit length, pound / inch \\
\hline SDOF & single degree of freedom \\
\hline $\mathrm{R}$ & radius, inches \\
\hline $\mathrm{r}$ & radial coordinate, inch \\
\hline St & static stress, psi \\
\hline $\mathrm{T}$ & time, second \\
\hline Tamb & ambient temperature, ${ }^{\circ}$ Rankine \\
\hline $\mathrm{t}$ & thickness, inch \\
\hline $\mathrm{u}$ & local axial coordinate, inch \\
\hline $\mathrm{V}_{0}$ & shock wave velocity, inch / second \\
\hline $\mathrm{V}_{\mathrm{cr}}$ & critical velocity, inch / second \\
\hline W & local radial coordinate, inch \\
\hline $\mathrm{x}$ & axial coordinate, inch \\
\hline $\mathrm{x}_{\mathrm{h}}$ & homogeneous solution \\
\hline $\mathrm{x}_{\mathrm{p}}$ & particular solution \\
\hline $\mathrm{z}$ & local radial coordinate, inch \\
\hline$\alpha$ & constant \\
\hline$\varepsilon$ & strain, inch / inch \\
\hline$\lambda$ & constant \\
\hline$\xi$ & constant \\
\hline$v$ & Poisson's ratio \\
\hline$\Phi$ & work ratio \\
\hline$\rho_{\text {fluid }}$ & fluid weight density, pound/ inch ${ }^{3}$ \\
\hline$\rho$ & pipe weight density, pound/ inch ${ }^{3}$ \\
\hline$\sigma$ & stress, psi \\
\hline$\sigma_{\mathrm{a}}$ & free vibration stress, psi \\
\hline$\sigma_{\mathrm{e}}$ & fatigue limit, psi \\
\hline$\sigma_{\mathrm{p}}$ & precursor stress, psi \\
\hline$\sigma_{\mathrm{ra}}$ & range stress, psi \\
\hline$\sigma_{\mathrm{s}}$ & step response stress, psi \\
\hline$\tau$ & velocity ratio \\
\hline$\theta$ & circumferential direction, radians \\
\hline$\omega$ & frequency, radians / second \\
\hline $\begin{array}{l}\omega_{\mathrm{n}} \\
\zeta_{\mathrm{f}}, \zeta_{\mathrm{S}}\end{array}$ & $\begin{array}{l}\text { natural frequency, radians / second } \\
\text { damping ratio }\end{array}$ \\
\hline$\Delta \mathrm{h}$ & change in enthalpy, BTU/ pound \\
\hline$\Delta \mathrm{s}$ & change in entropy, BTU/ pound ${ }^{\circ}$ Ranl \\
\hline
\end{tabular}




\section{INTRODUCTION}

Hoop stresses due to shock waves in pipes have received attention from numerous authors, but attention will be focused here on the work of a few. In particular, T. Simkins [1] presented work related to stresses in cannons when they were fired; Beltman, et. al. [2], presented work related to stresses in a gas filled tube subjected to a moving internal pressure wave; and work has also been presented to describe pipe stresses due to water hammer (Leishear [3]). All of this work is similar with respect to topic, in that hoop stresses, or breathing stresses, in a tube are created by a shock wave traveling along the bore of the tube at sonic or supersonic velocities. The fluids considered in the tubes may vary, but the fundamental equations are the same. The way in which these equations are handled by different authors results in different mathematical descriptions of the phenomena. Equations for are, for the most part, scattered throughout numerous references, and the purpose for this paper is to compile, clarify, discuss, and compare the different derivations in one source.

\section{ANALYSES}

The stresses in a tube, or pipe, are caused by the vibrations of the pipe wall as the shock travels through the pipe, as shown in Fig. 1. The shock wave travels at a velocity, $\mathrm{V}_{0}$, and a pressure increase occurs from $\mathrm{P}$ to $\mathrm{P}+\mathrm{P}_{0}$ across the shock. The initial pressure in the pipe, $\mathrm{P}$ is neglected in the remainder of this discussion. A precursor vibration occurs in front of the wave, and an aftershock vibration occurs in the wake of the shock. The strain jump is related to the median change in diameter of the pipe. The concept of flexural resonance is one approach to describe hoop stresses. The other approach is referred to as a step response. Both approaches are defined and discussed herein.

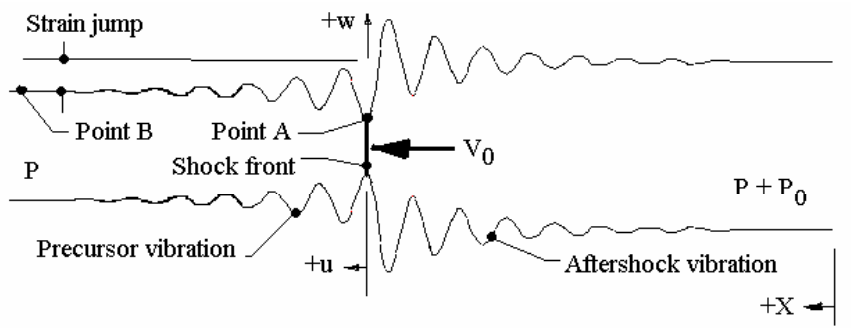

Figure 1: Pipe Wall Vibration

\section{Flexural Resonance in a Thin Walled Tube}

Simkins noted that Lame's equation (Eq. A37) as applied to gun barrel design was inadequate as projectile velocities increased, and he attributed the discrepancy to flexural resonance. The Lame equations assumed that hoop stresses could be calculated as static stresses using the pressure behind the projectile and available equations. However, Simkins noted that hoop stresses were a function of velocity, and that a maximum stress due to flexural resonance occurred at a critical velocity, $\mathrm{V}_{\text {cr }}$.

Critical Velocity. His derivation of the critical velocity for a thin walled tube is presented in App. A (Eq. A56) and is expressed as

$$
V_{\mathrm{cr}}=\sqrt{\frac{\mathrm{E} \cdot \mathrm{t} \cdot \mathrm{g}}{\rho \cdot \mathrm{R} \cdot \sqrt{3 \cdot\left(1-v^{2}\right)}}}
$$

An alternate equation (Eq. A61) for the critical velocity is

$$
V_{c r}=\sqrt{\frac{E \cdot t \cdot g}{\rho \cdot R \cdot \sqrt{3}}}
$$

The differences between these two equations are the assumed boundary conditions. The first equation assumes that the ends of the tube are free to move. The second equation assumes that both ends of the tube are restrained. Experimental observations showed that the predicted critical velocity needed to be corrected for applications to thick walled tubes, and Simkins' work [4] also provided those approximations.

Flexural Frequency. The frequency of vibrations (App. A) was determined at different velocities and was shown to be different before and after the shock, but at the critical velocity, the frequency before and after the shock equals (Eq. A58)

$$
\omega=\sqrt{\frac{2 \cdot E \cdot g}{\rho \cdot R^{2}}}
$$

Dynamic Load Factor. He also determined a dynamic load factor as shown in Fig. 2, which could be used to determine the maximum stress. To do so, the Lame equation was simply multiplied by the DLF to obtain the actual stress. Note that the stress is infinite at the critical velocity.

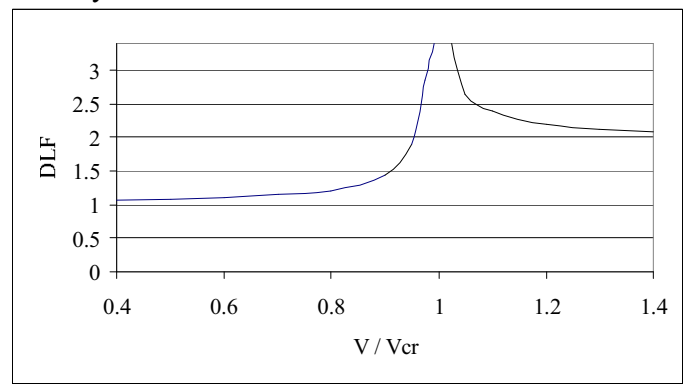

Figure 2: DLF's From Flexural Resonance Approximation (Simkins [1])

Step Response of a Thin Walled Tube to an Applied Shock

Recent work (Leishear [3] considered the problem from a different perspective, assuming that the pressure behind the shock was suddenly applied to a pipe wall as the shock arrived at a point on the pipe wall. This technique provided an approximation to assess the maximum stress due to a shock, and the derivation is presented in App. B. Essentially, the critical velocity provided by Beltman was assumed to be correct, but the pipe wall was assumed to vibrate at its natural frequency, rather than at the flexural frequency. Alternatively, the step response was considered to be independent of the critical velocity.

These assumptions are significantly different than the conclusions reached in a flexural resonance analysis.

Natural Frequency. The natural frequency of vibration for a thin walled tube with fixed ends was determined by Barez [5], such that

$$
\omega=\sqrt{\frac{\left(\frac{2 \cdot k}{(R) \cdot t}\right)+\left(\frac{E}{(R)^{2} \cdot\left(1-v^{2}\right)}\right)}{\rho+\frac{(R) \cdot \rho_{\text {fluid }}}{4 \cdot t}}} \cdot g
$$


Again, by changing the boundary condition at the pipe ends to a fixed end condition, the frequency can be written as

$$
\omega=\sqrt{\frac{\left(\frac{2 \cdot k}{(R) \cdot t}\right)+\left(\frac{E}{(R)^{2} \cdot(1)}\right)}{\rho+\frac{(R) \cdot \rho_{\text {fluid }}}{4 \cdot t}} \cdot g}
$$

The frequency is also affected by damping as discussed in App. B.

Dynamic Load Factor. The DLF's predicted by the step response solution are significantly different than those predicted using flexural resonance equations. The DLF was determined to have a maximum less than 4 at the critical velocity, as shown in Fig. 3.

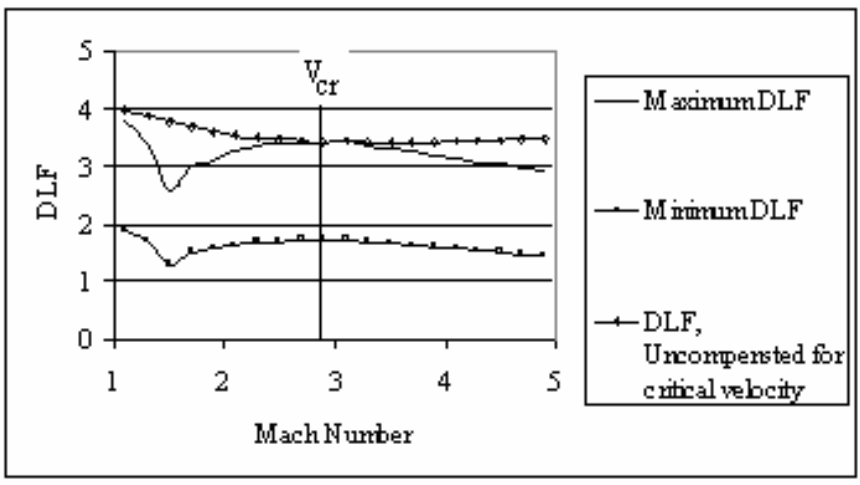

Figure 3: DLF's From Step Response Approximation

\section{Comparison of Equations to Experimental Data for a Thin Walled Tube}

Limited published data is available, and further research is certainly warranted. However, the available research provides significant insight into the mechanics of the problem to provide a comparison between the two approaches. The measures for comparison are frequency and DLF's. The work of Beltman, et. al., provided results for a gas filled aluminum tube subjected to a shock wave. Their testing provided results for a 1.027 inch diametergas filled, thin walled tube having a wall thickness of 0.063 inch.

Comparison of Frequencies. The precursor vibration frequencies were typically observed to be higher than the aftershock vibration frequencies as expected from the flexural resonance equations. However, the aftershock vibration frequency was better predicted by the step response equations, which predict the frequency to be less by a factor of the square root of 2 . The two theories predict different frequencies for the hoop stress. Again, further research is required to understand the frequency response.

DLF Comparison. The DLF at the critical velocity was experimentally observed to be a maximum of approximately 3.5 , when a DLF of infinity was predicted using the undamped flexural resonance equations. This disparity prompted the research leading to the step response equations. Also at velocities other than critical, the step response approach predicts higher DLF's than those predicted by assuming flexural resonance. An example at a speed significantly different than critical was considered for a thick walled tube

\section{Comparison of Equations to Experimental Data for a Thick Walled Tube}

Empirical data is available from two sources. In one case, the step response approach provided a reasonable estimate of the maximum strain in a thick walled cylinder (Leishear [3]), which considered a water hammer induced shock in a 2 NPS, schedule 40 pipe. Although the frequency was inaccurately predicted, the DLF of 2.2 was accurately predicted at $\mathrm{V}_{0} / \mathrm{V}_{\mathrm{cr}}=0.67$. Using Fig. 2, flexural resonance theory predicted the DLF $\approx 1.2$ from Fig. 2 .

The frequency for a long thick walled tube is expected to be a function of shock velocity, which may provide an alternative explanation for the differing frequencies across the shock. For a short thick walled tube, an FEA analysis showed that an eight inch long, 8 NPS, pipe vibrated at its natural frequency when subjected to an internal shock wave (Leishear [6]).

In the other case, Simkins [1] successfully compared detailed thick walled flexural resonance equations to experimental data for a gun barrel. In both cases, the frequencies were inadequately explained, and further research is required with respect to frequency.

\section{Prediction of the DLF Using the Step Response}

The maximum and minimum DLF's for a gas filled aluminum tube are shown in Fig. 2, and those DLF's depend on the critical velocity (Eq. B33). These maximum and minimum values occur at different points along a pipe subjected to a shock. In other words, the location of the measurement sensors affects the measured maximum strain. The maximum strain is also affected by end constraints of the tube.

Predicted DLF's uncompensated for the critical velocity are also shown in the figure (Eqs. B33 and B53). These alternative predictions are assumed to be higher than the actual DLF since simplifying assumptions were used with respect to frequencies in the step response technique. The differences associated with these assumptions can, once again, be resolved through further research. Regardless of frequency errors, a reasonable estimate of the DLF is provided near the critical speed, and a somewhat conservative overestimate of the DLF is provided at other velocities. The available research indicates that the predicted DLF's are, in fact, quite valid. As such, the step response should be a useful tool for the evaluation of hoop stresses on long pipes and tubes.

\section{CONCLUSION}

Dynamic load factors are required to determine the maximum stresses in tubes subjected to internal shocks, and the theory presented here provides an adequate prediction of the DLF. In particular, the derivations pertinent to this theory were presented to clarify the assumptions and solution techniques used to derive a theoretical approximation for the stresses. The concept of a dynamic load factor provides a multiplier to be used with available equations to predict the maximum stress during a dynamic event, such as the response to a shock wave traveling in a tube.

Approximations were made to derive this solution technique, and this technique was also compared to a previous solution, referred to as the flexural resonance technique. That solution is referred to as an exact technique, but it predicts the response to be infinite at the critical velocity, whereas the technique presented here accurately predicts the maximum strains at the critical velocity. There are still unanswered questions about the accuracy of either solution at velocities far removed from the critical velocity, since experimental data is unavailable at those velocities. There is also some question with respect to the step response solution and the data obtained by Beltman. The measured strains provided in their research agree with the step response predictions, but 
data summarized in their other graphs indicates that the flexural resonance theory may be more accurate. End effects and the limited number of sensors need further consideration.

\section{APPENDIX A: DERIVATION OF FLEXURAL RESONANCE EQUATIONS FOR A THIN WALLED TUBE}

There are two derivations for the critical velocity, depending on the assumptions with respect to the boundary conditions at the pipe ends. One derivation assumes the pipe ends to be fixed. The other derivation assumes one pipe ends to be unrestrained, or free.

\section{Flexural Resonance Assuming Free Pipe Ends}

The derivation for a tube with free ends was presented by Simkins, but is rewritten here to show all of the steps used in his analysis. Further details are available in the references, but the following derivation presents all of the equations used in the original referenced analyses.

Moment in a Differential Element. The response of a thin walled tube may be developed by first considering the bending of a differential element of the tube wall as shown in Fig. A1, where $\sigma_{\mathrm{x}}$ is the axial stress, $\mathrm{z}$ is measured from the center of a tube wall of thickness, $t, d z$ is the width of a differential element, and $M$ is the resultant moment due to bending of the tube wall.

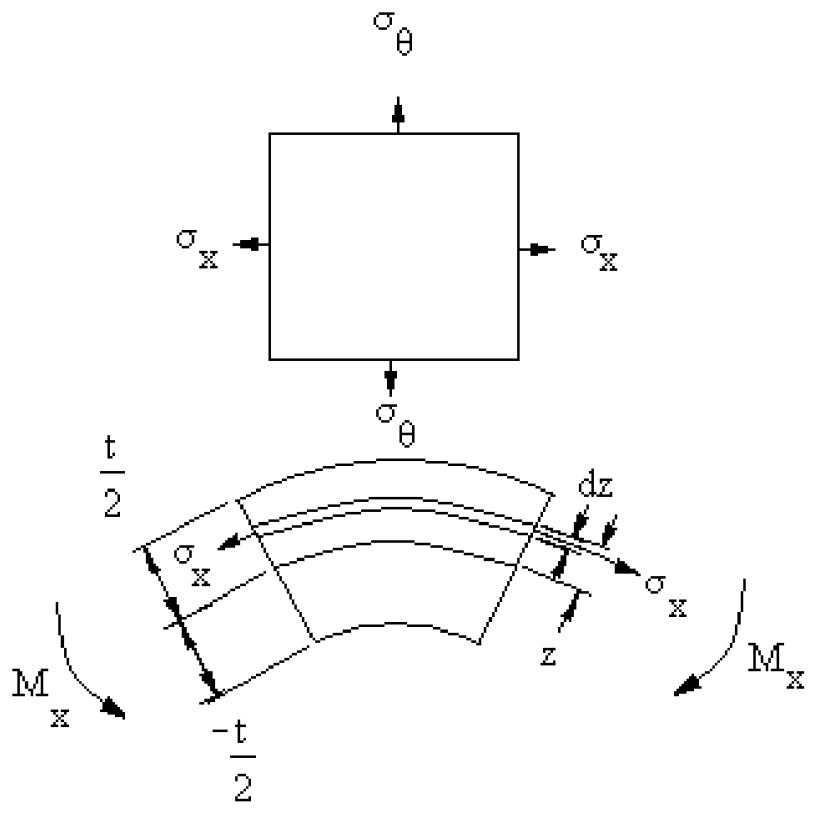

Figure A1: Differential Axial Element in the Tube Wall

The moment was determined as follows (Timoshenko and Woinowski-Krieger [7]). Using Hooke's Law for free end conditions

$$
\begin{gathered}
\varepsilon_{X}=\frac{\sigma_{X}}{E}-\frac{v \cdot \sigma_{\theta}}{E} \\
\varepsilon_{\theta}=\frac{\sigma_{\theta}}{E}-\frac{v \cdot \sigma_{X}}{E}=0
\end{gathered}
$$

where the local coordinate system in terms of $u$ and $w$ is shown in Fig. $1 ; \varepsilon_{\mathrm{x}}, \varepsilon_{\theta}, \sigma$, and $\sigma_{\theta}$, are the axial and hoop strains and stresses; $\mathrm{E}$ is the modulus of elasticity; and $v$ is Poisson's ratio. The curvature of the tube deflection equals
The unit elongation then equals

$$
-d^{2} w / d x^{2}
$$

$$
\varepsilon_{\mathrm{u}}=-\mathrm{z} \cdot \mathrm{d}^{2} \mathrm{w} / \mathrm{dx} \mathrm{x}^{2}
$$

where $u$ and $w$ are the axial and radial displacements respectively. On substitution,

$$
\begin{gathered}
\varepsilon_{X}=\frac{\left(1-v^{2}\right) \cdot \sigma_{X}}{E} \\
\sigma_{X}=\frac{E \cdot z}{\left(1-v^{2}\right)} \cdot \frac{d^{2} w}{d x^{2}}
\end{gathered}
$$

The bending moment is then calculated using the differential element.

$$
M_{X}=\int_{-t / 2}^{t / 2}\left(\sigma_{x} \cdot z\right) d z=\int_{-t / 2}^{t / 2}\left(\frac{E \cdot z^{2}}{\left(1-v^{2}\right)} \cdot \frac{d^{2} w}{d x^{2}}\right) d z=\frac{E \cdot t^{3}}{12 \cdot\left(1-v^{2}\right)} \cdot \frac{d^{2} w}{d x^{2}}
$$

and $\mathrm{M}_{\mathrm{x}}$ is expressed as

$$
M_{X}=\frac{M_{\theta}}{v}=-D \cdot \frac{d^{2} w}{d x^{2}}
$$

where $\mathrm{D}$ is the flexural rigidity, such that

$$
D=\frac{E \cdot t^{3}}{12 \cdot\left(1-v^{2}\right)}
$$

Membrane Forces in a Cylindrical Shell. Once the moment is determined for a differential element of the shell, the forces due to a statically applied pressure, P, can be determined using Fig. A2, where $\mathrm{Q}_{\mathrm{X}}$ is a shear force per unit length, and $\mathrm{N}_{\mathrm{x}}$ is a normal force per unit length, $M_{x}$ is the moment per unit length in the $x$-direction, $M_{\theta}$ is the moment per unit length in the tangential direction, $N_{\theta}$ is the circumferential force per unit length, $\mathrm{d} \theta$ is the differential length of the element along the circumference, $\mathrm{R}$ is the radius, and $\mathrm{dx}$ is the differential length of the element in the $\mathrm{x}$-direction.

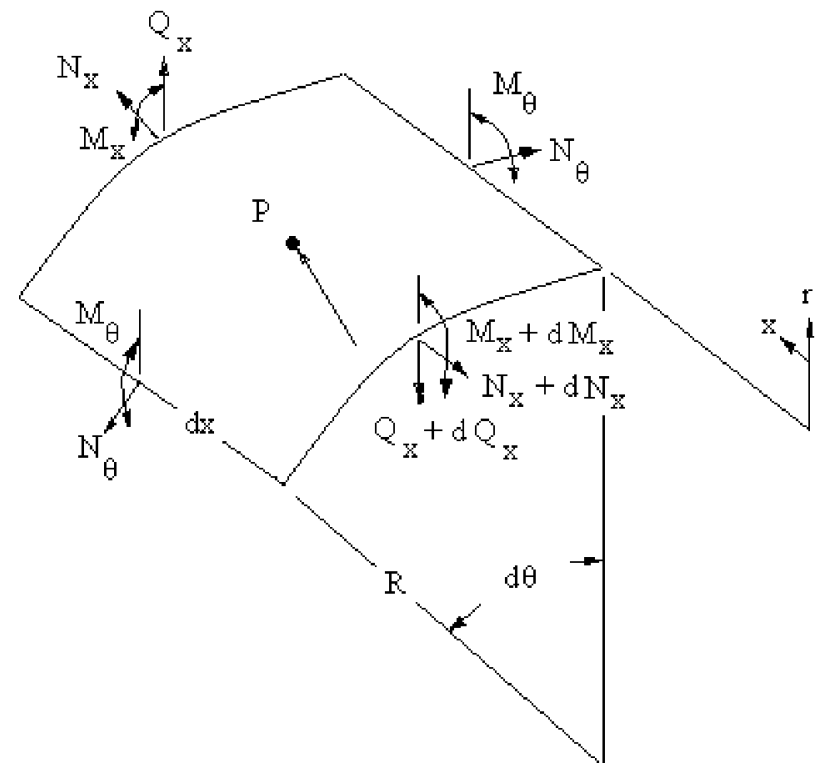

Figure A3: Differential Shell Element 
In the absence of axial loads (Timoshenko and Woinowski-Krieger [7]), the equilibrium equations for a shell are expressed as

$$
\begin{gathered}
\frac{d Q_{X}}{d x} \cdot R \cdot d x \cdot d \theta+N_{\theta} \cdot d x \cdot d \theta+P \cdot R \cdot d x \cdot d \theta=0 \\
\frac{d M_{X}}{d x} \cdot R \cdot d x \cdot d \theta-R \cdot Q_{X} \cdot d x \cdot d \theta=0
\end{gathered}
$$

These two equations are further reduced to

$$
\begin{gathered}
\frac{\mathrm{dQ}_{\mathrm{x}}}{\mathrm{dx}}+\frac{\mathrm{N}_{\theta}}{\mathrm{R}}+\mathrm{P}=0 \\
\frac{\mathrm{dM} \mathrm{x}}{\mathrm{dx}}-\mathrm{Q}_{\mathrm{x}}=0
\end{gathered}
$$

Assume that the strains equal

$$
\begin{aligned}
\varepsilon_{\mathrm{X}} & =\frac{\mathrm{du}}{\mathrm{dx}} \\
\varepsilon_{\theta} & =\frac{-\mathrm{w}}{\mathrm{R}}
\end{aligned}
$$

The normal force is then equal to

$$
\mathrm{N}_{\mathrm{X}}=\frac{\mathrm{E} \cdot \mathrm{t}}{1-\mathrm{v}^{2}} \cdot\left(\varepsilon_{\mathrm{X}}+v \cdot \varepsilon_{\mathrm{X}}\right)=\frac{\mathrm{E} \cdot \mathrm{t}}{1-\mathrm{v}^{2}} \cdot\left(\frac{\mathrm{du}}{\mathrm{dx}}-\frac{\mathrm{v} \cdot \mathrm{w}}{\mathrm{R}}\right)=0
$$

which yields

$$
\frac{\mathrm{du}}{\mathrm{dx}}=\frac{\mathrm{v} \cdot \mathrm{w}}{\mathrm{R}}
$$

On substitution, the hoop stress resultant equals

$$
\mathrm{N}_{\theta}=\frac{\mathrm{E} \cdot \mathrm{t}}{1-\mathrm{v}^{2}} \cdot\left(\varepsilon_{\theta}+v \cdot \varepsilon_{\mathrm{X}}\right)=\frac{\mathrm{E} \cdot \mathrm{t}}{1-\mathrm{v}^{2}} \cdot\left(\frac{-\mathrm{w}}{\mathrm{R}}+\frac{\mathrm{v} \cdot \mathrm{du}}{\mathrm{dx}}\right)
$$

The circumferential force is found to be

$$
\mathrm{N}_{\theta}=\frac{-\mathrm{E} \cdot \mathrm{t} \cdot \mathrm{w}}{\mathrm{R}}
$$

Axial Displacement in a Cylindrical Shell. The hoop stress resultant is used to determine the axial displacement of shell. Eliminating $\sigma_{\mathrm{rx}}$ from Eqs. A12 and A 13 yields

$$
\frac{\mathrm{d}^{2} \mathrm{M}_{\mathrm{x}}}{\mathrm{dx}^{2}}+\frac{\mathrm{N}_{\theta}}{\mathrm{R}}+\mathrm{P}=0
$$

Substitution of Eqs. A8 and A19 into A20 describes the axial displacement of a shell subjected to a pressure, $\mathrm{P}$, such that

$$
D \cdot \frac{d^{4} w}{d x^{4}}+\frac{E \cdot t \cdot w}{R^{2}}=P
$$

Equation of Motion for a Cylindrical Shell. The equation of motion for a cylinder can be determined with the aid of Eq. A12, which describes the radial forces on the shell. The radial forces per unit length on the shell element must equal the mass per unit length $(\rho \cdot t)$ times the radial acceleration of the shell wall $\left(\mathrm{d}^{2} \mathrm{w} / \mathrm{dx}^{2}\right)$, such that

$$
\frac{\partial\left(Q_{X}\right)}{\partial x}+\frac{N_{\theta}}{R}+P=\rho / g \cdot t \cdot \frac{\partial^{2} w}{\partial T^{2}}
$$

On substitution of Eqs. A8, A13 and A19, Eq. A22 yields

$$
\frac{\mathrm{D} \cdot \partial^{4} \mathrm{w}}{\partial \mathrm{x}^{4}}+\frac{\mathrm{E} \cdot \mathrm{t} \cdot \mathrm{w}}{\mathrm{R}^{2}}+\frac{\rho / \mathrm{g} \cdot \mathrm{t} \cdot \partial^{2} \mathrm{w}}{\partial \mathrm{T}^{2}}=\mathrm{P}\left(1-\mathrm{H}\left(\mathrm{x}-\mathrm{V}_{0} \cdot \mathrm{T}\right)\right)
$$

where $\mathrm{T}$ is the time; $\mathrm{V}_{0}$ is the velocity of the coordinate system, or shock wave in this case; and $\mathrm{H}$ is the Heaviside step function expressing the pressure as a function of time. By a change of variables Eq. A23 is referenced to a moving coordinate system using the chain rule for differential equations, such that

$$
\begin{aligned}
& \frac{\mathrm{D} \cdot \partial^{4} \mathrm{w}}{\partial(\mathrm{x}-\mathrm{V} \cdot \mathrm{T})^{4}} \cdot \frac{\partial^{4}\left(\mathrm{x}-\mathrm{V}_{0} \cdot \mathrm{T}\right)}{\partial \mathrm{x}^{4}}+\frac{\mathrm{E} \cdot \mathrm{t} \cdot \mathrm{w}}{\mathrm{R}^{2}}+\frac{\rho / \mathrm{g} \cdot \mathrm{t} \cdot \partial^{2} \mathrm{w}}{\partial(\mathrm{x}-\mathrm{V} \cdot \mathrm{T})^{2}} \cdot \frac{\partial^{2}\left(\mathrm{x}-\mathrm{V}_{0} \cdot \mathrm{T}\right)}{\partial \mathrm{T}^{2}}= \\
& \mathrm{P} \cdot\left(1-\mathrm{H}\left(\mathrm{x}-\mathrm{V}_{0} \cdot \mathrm{T}\right)\right)
\end{aligned}
$$

Simplifying,

$$
\text { where } \begin{aligned}
\frac{d^{4} w}{d \xi^{4}}+w+\frac{2 \cdot \lambda \cdot d^{2} w}{d \xi^{2}}=P\left(1-H\left(x-V_{0} \cdot T\right)\right. \\
\gamma=\sqrt[4]{\frac{E \cdot t}{R^{2} \cdot D}} \\
\xi=\gamma \cdot\left(x-V_{0} \cdot T\right) \\
2 \cdot \lambda=\rho / g \cdot V_{0}^{2} \sqrt{\frac{R^{2}}{E \cdot t \cdot D}}
\end{aligned}
$$

Evaluation of Flexural Resonance. Equation A25 is the basis of the following evaluation of flexural resonance provided by Simkins [1]. This equation was the beginning of his analysis. The equation is rewritten in terms of displacements before and after the shock wave.

$$
\begin{gathered}
\frac{d^{4} w^{(1)}}{d \xi^{4}}+w(1)+\frac{2 \cdot \lambda \cdot d^{2} w^{(1)}}{d \xi^{2}}=\frac{P_{0} \cdot R^{2}}{E \cdot t} \\
\frac{d^{4}{ }^{(2)}}{d \xi^{4}}+w^{(2)}+\frac{2 \cdot \lambda \cdot d^{2} w^{(2)}}{d \xi^{2}}=0
\end{gathered}
$$

where the subscripts (2) and (1) indicate the displacements before and after the shock respectively.

To further consider the displacement, assume that Eq. A29 has a solution of the form

$$
\lim _{\alpha \rightarrow 0} \frac{\mathrm{R}^{2} \cdot \mathrm{P}_{0} \cdot \mathrm{e}^{\mathrm{i} \cdot \alpha \cdot \xi}}{\mathrm{E} \cdot \mathrm{t}}=\lim _{\alpha \rightarrow 0}\left(\alpha^{4}-2 \cdot \lambda \cdot \alpha^{2}+1\right) \cdot \mathrm{A} \cdot \mathrm{e}^{\mathrm{i} \cdot \alpha \cdot \xi}
$$

where $\mathrm{w}^{(1)}=\mathrm{A} \cdot \mathrm{e}^{\mathrm{i} \cdot \dot{\alpha} \hat{\xi} \xi}$. For the homogeneous solution, let

$$
\begin{gathered}
\alpha^{4}-2 \cdot \lambda \cdot \alpha^{2}+1=0 \\
\alpha^{2}=\lambda \pm \sqrt{\lambda^{2}-1}
\end{gathered}
$$

Then the four roots of Eq. A32 are

$$
\begin{gathered}
\alpha= \pm \frac{(\mathrm{i} \sqrt{1-\lambda} \pm \sqrt{1+\lambda})}{\sqrt{2}}= \pm \mathrm{c} \pm \mathrm{d} \cdot \mathrm{i} \\
\mathrm{d}=\sqrt{1-\lambda} \\
\mathrm{c}=\sqrt{1+\lambda}
\end{gathered}
$$

By inspection, the particular solution, $\mathrm{w}_{\mathrm{p}}$ for Eqs. A29 is expressed as Lame's equation for a cylinder, such that

$$
w_{p}=\frac{P_{0} \cdot R^{2}}{E \cdot t}
$$

Similarly, the roots of the homogeneous solution to Eq. A30 are the same as those listed in Eq. A34, and the particular solution is

$$
\mathrm{w}_{\mathrm{p}}=0
$$

The general solutions to Eqs. A29 and A30 can then be written as 


$$
\begin{aligned}
& { }^{(1)}= \\
& \mathrm{A}_{1} \mathrm{e}^{(\mathrm{ic}+\mathrm{d}) \cdot \xi}+\mathrm{A}_{2} \mathrm{e}^{-(\mathrm{ic}+\mathrm{d}) \xi}+\mathrm{A}_{3} \cdot \mathrm{e}^{(\mathrm{ic}-\mathrm{d}) \cdot \xi}+\mathrm{A}_{4} \mathrm{e}^{(-\mathrm{ic}-\mathrm{d}) \cdot \xi}+\mathrm{w}_{\mathrm{p}} \\
& { }_{\mathrm{W}}^{(2)}=\mathrm{A} 5 \cdot \mathrm{e}^{(\mathrm{i} \cdot \mathrm{c}+\mathrm{d}) \cdot \xi}+\mathrm{A}_{6} \cdot \mathrm{e}^{-(\mathrm{i} \cdot \mathrm{c}+\mathrm{d}) \cdot \xi}+\mathrm{A}_{7} \cdot \mathrm{e}^{(\mathrm{i} \cdot \mathrm{c}-\mathrm{d}) \xi} \\
& \quad+\mathrm{A}_{8} \cdot \mathrm{e}^{(-\mathrm{i} \cdot \mathrm{c}-\mathrm{d}) \cdot \xi}
\end{aligned}
$$

The boundary conditions to solve these equations assume continuity at the shock, where $\xi=0$, and are stated as

Displacement continuity, $\mathrm{w}^{(1)}-\mathrm{w}^{(2)}=0$

Rotational continuity, $\frac{\mathrm{dw}(1)}{\mathrm{d} \xi}-\frac{\mathrm{dw}(2)}{\mathrm{d} \xi}=0$

Moment continuity, $\quad \frac{\mathrm{d}^{2} \mathrm{w}^{(1)}}{\mathrm{d} \xi^{2}}-\frac{\mathrm{d}^{2} \mathrm{w}^{(2)}}{\mathrm{d} \xi^{2}}=0$

Shear continuity, $\quad \frac{\mathrm{d}^{3} \mathrm{w}^{(1)}}{\mathrm{d} \xi^{3}}-\frac{\mathrm{d}^{3} \mathrm{w}^{(2)}}{\mathrm{d} \xi^{3}}=0$

Velocities dictate three different solutions to these equations. Below the critical velocity, $\lambda<1$; at the critical velocity, $\lambda=1$; and above the critical velocity $\lambda>1$ To reduce the number of equations, the boundary condition at the ends of the pipe were considered.

Below the critical velocity as $x \rightarrow \pm \infty, \xi \rightarrow \pm \infty$, the exponents in Eqs. A39 and A40 must be negative to have bounded solutions. Therefore when $\lambda<1, \mathrm{~A}_{3}=\mathrm{A}_{4}=\mathrm{A}_{5}=\mathrm{A}_{6}=0$ and

$$
\begin{gathered}
\mathrm{w}^{(1)}=\mathrm{A}_{1} \mathrm{e}^{(\mathrm{ic}+\mathrm{d}) \xi \xi}+\mathrm{A}_{2} \mathrm{e}^{-(\mathrm{ic}+\mathrm{d}) \cdot \xi}+\mathrm{w}_{\mathrm{p}} \\
{ }_{\mathrm{W}}{ }^{(2)}=\mathrm{A}_{7} \cdot \mathrm{e}^{(\mathrm{i} \cdot \mathrm{c}-\mathrm{d}) \cdot \xi}+\mathrm{A}_{8} \cdot \mathrm{e}^{(-\mathrm{i} \cdot \mathrm{c}-\mathrm{d}) \cdot \xi}
\end{gathered}
$$

Substituting the boundary conditions of Eqs. A41 -A44 into Eqs. A39 and $\mathrm{A} 40$ yields

$$
\left|\begin{array}{l}
A_{1}+A_{2}-A_{7}-A_{8}=-\frac{P_{0} \cdot R^{2}}{E \cdot t} \\
\alpha_{1} \cdot A_{1}+\alpha_{2} \cdot A_{2}-\alpha_{3} \cdot A_{7}-\alpha_{4} \cdot A_{8}=0 \\
\alpha_{1}^{2} \cdot A_{1}+\alpha_{2}^{2} \cdot A_{2}-\alpha_{2}^{2} \cdot A_{7}-\alpha_{4}^{2} \cdot A_{8}=0 \\
\alpha_{1}^{3} \cdot A_{1}+\alpha_{2}^{3} \cdot A_{2}-\alpha_{2}^{3} \cdot A_{7}-\alpha_{4}^{3} \cdot A_{8}=0
\end{array}\right|
$$

Solving A45 - A47,

$$
{ }_{W}^{(1)}=\frac{P_{0} \cdot R^{2} \cdot e^{d \cdot \xi}}{2 \cdot E \cdot t}-\left(-\cos (c \cdot \xi)+\frac{d^{2}-c^{2}}{2 \cdot c \cdot d}-\sin (c \cdot \xi)\right)+1
$$

for $\xi \leq 0$ behind the shock, and

${ }_{w}^{(2)}=\frac{P_{0} \cdot R^{2}}{E \cdot t} \cdot w^{(1)}=\frac{P_{0} \cdot R^{2} \cdot e^{d} \cdot \xi}{2 \cdot E \cdot t}-\left(\cos (c \cdot \xi)+\frac{d^{2}-c^{2}}{2 \cdot c \cdot d}-\sin (c \cdot \xi)\right)$

for $\xi \geq 0$ in front of the shock.

At the critical velocity $\lambda=0, d=0$ and a solution similar to Eq. A47 is nonexistent since

$$
|\operatorname{coef}|=0
$$

Above the critical velocity $\lambda>1$, and Simkins solved the pertinent equations to show that

$$
\mathrm{w}^{(1)}=\frac{\mathrm{P}_{0} \cdot \mathrm{R}^{2}}{2 \cdot \mathrm{E} \cdot \mathrm{t}} \cdot\left(\frac{\mathrm{b}^{2} \cdot \cos (\mathrm{a} \cdot \xi)}{\mathrm{b}^{2}-\mathrm{a}^{2}}+1\right)
$$

$$
\begin{aligned}
\mathrm{w}^{(2)} & =\frac{\mathrm{P}_{0} \cdot \mathrm{R}^{2}}{2 \cdot \mathrm{E} \cdot \mathrm{t}} \cdot\left(\frac{-\mathrm{a}^{2} \cdot \cos (\mathrm{b} \cdot \xi)}{\mathrm{b}^{2}-\mathrm{a}^{2}}\right) \\
\mathrm{a} & =\sqrt{\frac{1+\lambda}{2}}-\sqrt{\frac{\lambda-1}{2}} \\
\mathrm{~b} & =\sqrt{\frac{1+\lambda}{2}}+\sqrt{\frac{\lambda-1}{2}}
\end{aligned}
$$

Equations A48 - A52 describe the tube wall motion.

Dynamic Load factor. Figure 1 was drawn by defining the DLF as

$$
\mathrm{DLF}=\frac{\mathrm{w}}{\left(\frac{\mathrm{P}_{0} \cdot \mathrm{R}^{2}}{2 \cdot \mathrm{E} \cdot \mathrm{t}}\right)}
$$

Critical velocity. Once all of the equations were developed, the critical velocity and frequency were determined. The critical velocity occurs at $\lambda=0$, and Eq. A28 yields

$$
V_{c r}=\sqrt{\frac{E \cdot t \cdot g}{\rho \cdot R \cdot \sqrt{3 \cdot\left(1-v^{2}\right)}}}
$$

Breathing Mode Frequency. Inspection of Eq. A27 shows that the frequency seen by a stationary observer equals

$$
\omega=\mathrm{V} \cdot \gamma
$$

At the critical velocity, Eqs. A56 and A57 yield

$$
\omega=\sqrt{\frac{2 \cdot E \cdot g}{\rho \cdot R^{2}}}
$$

Simkins noted that the critical velocity and frequencies were significantly different for thick walled tubes, and the reader is referred to his work for further details. For the purposes of this paper, this thin wall solution was compared to the step response solution.

\section{Flexural Resonance Assuming Fixed Pipe Ends}

The analysis for a pipe with fixed ends is identical to that for free ends, except that the initial boundary conditions are different. Experimentally, the axial and hoop strains were shown to be (Leishear [8]) equal, such that

$$
\varepsilon_{\mathrm{X}}=\varepsilon_{\theta}
$$

Reconsidering Eqs. A1 and A2, the axial and hoop stresses must also be equal, such that

$$
\sigma_{\mathrm{X}}=\sigma_{\theta}
$$

By substitution into Eqs. A3 - A56, the critical velocity for a fixed end pipe equals

$$
V_{\mathrm{cr}}=\sqrt{\frac{\mathrm{E} \cdot \mathrm{t} \cdot \mathrm{g}}{\rho \cdot \mathrm{R} \cdot \sqrt{3 \cdot(1)}}}
$$

Essentially, the $v^{2}$ term is dropped from all of the equations. For typical structural materials, where $v=0.3$, the difference in the critical velocity due to end restraints of the tube is approximately only 5 percent. Frequencies are the same for either end constraint, as shown by substitution of the appropriate terms into Eq. A57. 


\section{APPENDIX B: DERIVATION OF THE STEP RESPONSE EQUATIONS}

Similar to the flexural resonance derivation, the equations describing the step response derivation are scattered throughout numerous references. Again, the intent here is to provide the requisite equations to provide an adequate review of the analysis technique. All of the referenced equations have been published, and further details are available in the applicable references. Figure 1 shows the problem to be addressed. Essentially, the step response technique assumes that the response of a shell can be calculated for a suddenly applied load, and that this dynamic load is continually applied to the tube wall at the velocity of the shock wave, $\mathrm{V}$. To start the discussion, a suddenly applied load at $\mathrm{V}=0$ is considered, using Figs. B1 and B2. Through wall radial stresses are excluded from this discussion.

The stresses due to expansion of the tube, or breathing stresses, are the focus of this discussion. An isotropic tube is assumed to act as a single degree of freedom (SDOF) oscillator subjected to a suddenly applied step increase in pressure as shown in Fig. B1. T evaluate the dynamic stresses, the static stress is first required followed by the equation of motion. Once these equations are presented the dynamics of the step pressure moving at a sonic velocity are discussed below.

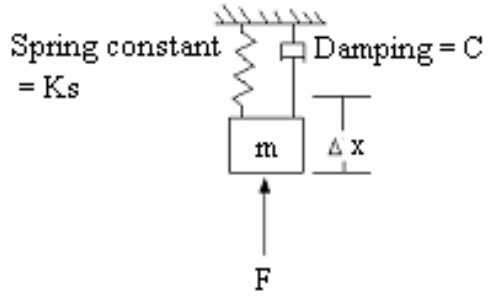

Figure B1: SDOF System with Excitation

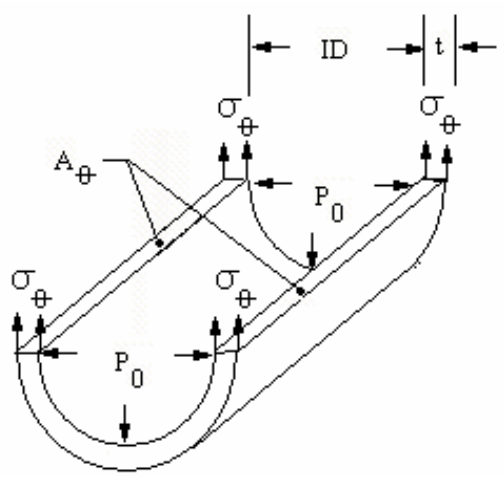

Figure B2: SDOF Pipe Model

\section{Static Stress}

The static Lame stress is determined by assuming a unit length subjected to a pressure, $P_{0}$, with opposing hoop stresses equal to $2 \cdot \sigma_{\sigma}$. This thin wall approximation stress is expressed as

$$
\sigma_{\theta}=\mathrm{P}_{0} \cdot \frac{\mathrm{R}}{\mathrm{t}}
$$

A more informative form of the hoop stress is the thick wall approximation, which can be found as follows (Lubliner [9]).

$$
\varepsilon_{\mathrm{R}}=\frac{\mathrm{d}\left(\mathrm{R} \varepsilon_{\theta}\right)}{\mathrm{dR}}=\frac{\sigma_{\mathrm{R}}-v \cdot \sigma_{\theta}}{\mathrm{E}}
$$

$$
\varepsilon_{\theta}=\frac{\sigma_{\theta}-v \cdot \sigma_{R}}{E}
$$

On substitution,

$$
\frac{\mathrm{d}\left((1-v) \cdot \sigma_{\theta}-v_{\mathrm{R}}\right)}{\mathrm{dR}}=\frac{\sigma_{\mathrm{R}}-\sigma_{\theta}}{\mathrm{E}}
$$

By symmetry,

$$
\frac{\mathrm{d} \sigma_{\mathrm{R}}}{\mathrm{dR}}+\frac{\sigma_{\mathrm{R}}-\sigma_{\theta}}{\mathrm{R}}=0
$$

Substituting Eq. B4 into B5 yields

$$
\frac{\mathrm{d}\left(\sigma_{\theta}-\sigma_{\mathrm{R}}\right)}{\mathrm{dR}}+\frac{2 \cdot\left(\sigma_{\theta}-\sigma_{\mathrm{R}}\right)}{\mathrm{R}}=0
$$

which can be solved by assuming two constants $A_{1}$ and $A_{2}$ such that

$$
\begin{aligned}
& \sigma_{\theta}-\sigma_{\mathrm{R}}=\frac{2 \cdot \mathrm{A}_{2}}{\mathrm{R}^{2}} \\
& \text { and } \quad \sigma_{\theta}+\sigma_{\mathrm{R}}=2 \cdot \mathrm{A}_{1} \\
& \sigma_{\theta}=-\frac{\left(\left.\sigma_{\mathrm{R}}\right|_{\mathrm{R}=\mathrm{ID}}\right)}{2}+\frac{\left.\sigma_{\mathrm{R}}\right|_{\mathrm{R}=\mathrm{ID}}}{2 \cdot\left(1-\left(\frac{\mathrm{ID}}{\mathrm{OD}}\right)^{2}\right)} \cdot\left(1+\left(\frac{\mathrm{ID}}{\mathrm{OD}}\right)^{2}+2 \cdot\left(\frac{\mathrm{ID}}{\mathrm{R}}\right)^{2}\right)
\end{aligned}
$$

which simplifies to

$$
\sigma_{\theta}=\mathrm{P}_{0} \cdot \frac{\mathrm{ID}^{2} \cdot\left(\mathrm{OD}^{2}+\mathrm{ID}^{2}\right)}{\mathrm{R}^{2} \cdot\left(\mathrm{OD}^{2}-\mathrm{ID}^{2}\right)}
$$

Therefore, the maximum stress occurs at the inside diameter, such that

$$
\mathrm{St}=\sigma_{\theta}=\mathrm{P}_{0} \cdot \frac{\left(\mathrm{OD}^{2}+\mathrm{ID}^{2}\right)}{\left(\mathrm{OD}^{2}-\mathrm{ID}^{2}\right)}
$$

where St is the static hoop stress, and $\sigma_{\theta}$ is considered to be the dynamic hoop stress for the remainder of this paper. The use of St is provided to prevent some confusion between the static and dynamic expressions for the hoop stress. Having established the static hoop stress for the cylinder, this stress needed to be related to the equation of motion.

\section{Equation of Motion for a SDOF Oscillator}

The equation of motion for a cylinder may be derived from the general equation of motion for a SDOF system as shown in Fig. B1, and can be expressed as

$$
\frac{\mathrm{d}^{2}(\mathrm{~m} \cdot \mathrm{x})}{\mathrm{dT}^{2}}+\frac{\mathrm{d}(\mathrm{C} \cdot \mathrm{x})}{\mathrm{dT}}+\mathrm{K} \cdot \mathrm{x}=\mathrm{F}(\mathrm{T})
$$

The response of a SDOF oscillator shown in Fig. B1 is available (Thomson [10]) and is derived as follows. When $\mathrm{F}(\mathrm{T})=\mathrm{F}=\mathrm{a}$ constant,

$\frac{\mathrm{d}^{2}(\mathrm{x})}{\mathrm{dT}^{2}}+\frac{\mathrm{d}(\mathrm{C} \cdot \mathrm{x})}{\mathrm{dT}}+\mathrm{K} \cdot \mathrm{x}=\frac{\mathrm{d}^{2}(\mathrm{~m} \cdot \mathrm{x})}{\mathrm{dT}^{2}}+\frac{2 \cdot \zeta \cdot \omega \cdot \mathrm{d}(\mathrm{x})}{\mathrm{dT}}+\omega^{2} \cdot \mathrm{x}=\frac{\mathrm{F}}{\mathrm{m}}(\mathrm{B} 13)$

where the frequency and damping ratio equal

$$
\begin{gathered}
\omega=\sqrt{\frac{\mathrm{K}}{\mathrm{m}}} \\
\zeta=\frac{\mathrm{C}}{2 \cdot \sqrt{\mathrm{K} \cdot \mathrm{m}}}
\end{gathered}
$$


Homogeneous Solution to the Equation of Motion. The homogeneous solution to Eq. B13 is

$$
\frac{\mathrm{d}^{2}(\mathrm{~m} \cdot \mathrm{x})}{\mathrm{dT}^{2}}+\frac{\mathrm{d}(\mathrm{C} \cdot \mathrm{x})}{\mathrm{dT}}+\mathrm{K} \cdot \mathrm{x}=0
$$

Assuming

$$
\mathrm{x}=\mathrm{e}^{\mathrm{s} \cdot \mathrm{T}}
$$

where $\mathrm{s}$ is a constant substituting Eq. B17 into Eq. B16 yields

$$
\left(\mathrm{m} \cdot \mathrm{s}^{2}+\mathrm{C} \cdot \mathrm{s}+\mathrm{K}\right) \cdot \mathrm{e}^{\mathrm{s} \cdot \mathrm{T}}=0
$$

which has two roots equal to

$$
\mathrm{s}_{1,2}=\left(-\zeta \pm \sqrt{\zeta^{2}-1}\right) \cdot \omega
$$

When the motion is oscillatory, $\zeta \leq 1$, the general solution to Eq. $\mathrm{B} 6$ is expressed as

$$
\begin{aligned}
x_{h} & =A_{1} \cdot e^{s_{1} \cdot T}+A_{2} \cdot e^{-s_{2} \cdot T} \\
& =e^{-\zeta \cdot \omega \cdot T} \cdot\left(A_{1} \cdot e^{i \cdot \omega \cdot T \sqrt{1-\zeta^{2}}}+A_{2} \cdot e^{-i \cdot \omega \cdot T} \sqrt{1-\zeta^{2}}\right) \\
& =A_{4} \cdot e^{-\zeta \cdot \omega \cdot T} \cdot \sin \left(\sqrt{1-\zeta^{2}} \cdot \omega \cdot T+A_{5}\right)
\end{aligned}
$$

Particular Solution to the Equation of MotionThe particular solution is found, using the method of undetermined coefficients. Assuming a solution of the form

$$
x_{p}=A_{5} \cdot \sin (\omega \cdot T)+A 6 \cdot \cos (\omega \cdot T)
$$

and substituting $\mathrm{X}_{\mathrm{p}}$ (Eq. B21) into Eq B13 and differentiating yields

$$
\begin{aligned}
x_{p}= & -A_{5} \cdot \omega^{2} \cdot \sin (\omega \cdot T)-A_{6} \cdot \omega^{2} \cdot \cos (\omega \cdot T) \\
& +2 \cdot \zeta \cdot \omega \cdot\left(\omega \cdot A_{5} \cdot \cos (\omega \cdot T)-\omega \cdot A_{6} \cdot \sin (\omega T)\right) \\
& +\omega^{2} \cdot\left(A_{5} \cdot \sin \left(\omega \cdot T+A_{6} \cdot \cos (\omega \cdot T)+A_{7}\right)\right)=\frac{F}{m}
\end{aligned}
$$

Using the boundary conditions,

$$
\text { at } \mathrm{T}=0, \mathrm{x}=0 \text { and } \frac{\mathrm{dx}}{\mathrm{dT}}=0
$$

like coefficients of Eq. B22 can be determined such that

$$
\begin{gathered}
\mathrm{A}_{7}=\frac{\mathrm{F}}{\mathrm{m} \cdot \omega^{2}}=\frac{\mathrm{F}}{\mathrm{K}} \\
\mathrm{A}_{5}=\mathrm{A}_{6}=0
\end{gathered}
$$$$
\text { Then } \quad x_{p}=\frac{F}{K}
$$

General Solution to the Equation of Motion for a Step Response. The general solution is found by adding the homogeneous and particular solutions (Eq. B20 + Eq. B26), and substituting the boundary conditions (B23) to find the constants $\mathrm{A}_{4}$ and $\mathrm{A}_{5}$. The general solution for the response to a suddenly applied force is then expressed as

$$
x(T)=\frac{F}{K} \cdot\left(1-\frac{\mathrm{e}^{-\zeta \cdot \omega \cdot T}}{\sqrt{1-\zeta^{2}}} \cdot \cos \left(\mathrm{T} \cdot \omega \cdot \sqrt{1-\zeta^{2}}\right)-\left(\frac{\zeta}{\sqrt{1-\zeta^{2}}}\right)\right)
$$

\section{Equation of Motion for a Cylinder Subjected to a Sudden Internal Pressure}

Having defined the step response for a SDOF oscillator, a cylinder needed to be described in terms of this response. Returning to Fig. B2, the variable, $\sigma_{\theta}$, can be expressed in terms of $\mathrm{x}$ by a change of variables.

To change the variables, the circumference of the pipe is compared to the SDOF system using Hooke's Law such that,

$$
\mathrm{F}_{\mathrm{x}}=\mathrm{K} \cdot \Delta \mathrm{x}=\mathrm{K} \cdot \mathrm{L} \cdot \varepsilon_{\mathrm{x}}
$$

where $\mathrm{L}$ is the length of the spring, and $\varepsilon_{\mathrm{x}}=\Delta \mathrm{x} / \mathrm{L}$. Similarly, the circumference, $\mathrm{c}$, increases by a length equal to $\Delta \mathrm{c}$ when it expands. The static circumferential force expands the pipe wall with a force equal to

$$
\mathrm{F}_{\theta}=\mathrm{K} \cdot \Delta \mathrm{c}=\mathrm{K} \cdot \mathrm{c} \cdot \varepsilon_{\theta}=\mathrm{K} \cdot \mathrm{c} \cdot \mathrm{St} / \mathrm{E}
$$

where $\varepsilon_{\theta}=\Delta \mathrm{c} / \mathrm{c}$. From the basic definition of a static stress

$$
\mathrm{F}_{\theta}=\mathrm{St} \cdot \mathrm{A}_{\theta}
$$

Assuming a unit area, substituting Eqs. B11, and B30 into B29, and using Hooke's Law, and equating $\mathrm{F}=\mathrm{P}_{0} / \mathrm{A}$

$$
\begin{aligned}
& \sigma(\mathrm{T})=\mathrm{St} \cdot\left(1-\frac{\mathrm{e}^{-\zeta \cdot \omega \cdot \mathrm{T}}}{\sqrt{1-\zeta^{2}}} \cdot \cos \left(\mathrm{T} \cdot \omega \cdot \sqrt{1-\zeta^{2}}\right)-\mathrm{a} \tan \left(\frac{\zeta}{\sqrt{1-\zeta^{2}}}\right)\right. \\
& \sigma_{\mathrm{S}}(\mathrm{T})=\mathrm{P}_{0} \cdot \frac{\left(\mathrm{OD}^{2}+\mathrm{ID}^{2}\right)}{\left(\mathrm{OD}^{2}-\mathrm{ID}^{2}\right)} \cdot \mathrm{H}\left(\mathrm{T}-\frac{\mathrm{x}}{\mathrm{V}_{0}}\right) \\
& \cdot\left(1-\frac{\mathrm{e}^{-\zeta \cdot \omega \mathrm{T}}}{\sqrt{1-\zeta^{2}}} \cdot \cos \left(\mathrm{T} \cdot \omega \cdot \sqrt{1-\zeta^{2}}\right)-\operatorname{atan}\left(\frac{\zeta}{\sqrt{1-\zeta^{2}}}\right)\right)(\mathrm{B} 32)
\end{aligned}
$$

where $\mathrm{H}$ is the Heaviside step function, used to describe the response as the shock arrives at a point at time $\mathrm{x} / \mathrm{V}_{0}$. This equation describes the stresses in the pipe when the entire inner pipe wall is subjected to a suddenly applied pressure, and is appropriate to predict the maximum response for short pipes (Leishear [6]). However, the response of a long pipe is much more complex due to the moving pressure discontinuity at the shock wave, which induces the pipe stresses.

\section{Pipe Stresses Due to a Shock Wave}

Referring to Fig. 1, the response of the pipe wall is assumed to consist of a precursor vibration and an aftershock vibration. Furthermore, the aftershock vibration is assumed to consist of two separate vibrations. Consequently, three vibrations need consideration.

First of all, one vibration is due to the step response after the shock. Stresses, $\sigma_{\mathrm{S}}$, are assumed to act in accordance with Eq. B32 as the shock moves at a sonic, or supersonic, velocity in the tube. The pipe is assumed to vibrate at its damped natural frequency in response to an excitation force.

Second, a precursor free vibration stress, $\sigma_{\mathrm{P}}$, due to the step response is assumed. If the wall vibrates behind the shock, the wall in front of the shock must also vibrate in response to the applied step pressure to maintain continuity. However, the vibration must be a free vibration since an applied load on the pipe wall in front of the shock is nonexistent.

Third, a free vibration stress, $\sigma_{\mathrm{a}}$, is also assumed to exist as part of the aftershock vibration. This vibration is assumed to exist due to the motion of the shock. If a discontinuous step pressure is applied to the pipe wall and the shock is not moving $(\mathrm{V}=0)$, both the step response and precursor vibration exist. However, the shock is moving, and as such an observer at the shock experiences an additional effect. As he moves into the precursor vibration, the wall is already vibrating, and 
this additional vibration needs to be added to the other two vibration induced stresses, such that the total vibration is described as

$$
\sigma(\mathrm{T})=\sigma_{\mathrm{p}}+\sigma_{\mathrm{s}}+\sigma_{\mathrm{a}}
$$

The stress due to the step response is presented in Eq. B32, but the other two stresses require further discussion.

\section{Precursor Stresses}

The precursor stresses are better visualized if damping is excluded from the equations of motion. For example Eq. B33 is simplified to

$$
\sigma(\mathrm{T})=\mathrm{St} \cdot(1-\cos (\omega \cdot \mathrm{T}))
$$

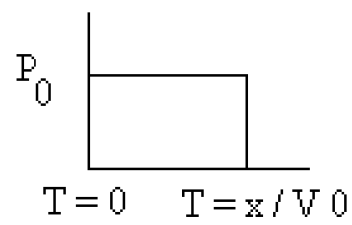

\section{Figure B3: Free Vibration Model}

Consider Fig. B3 to evaluate precursor free vibrations due to a step response. One vibration occurs at an arbitrary time, $\mathrm{T}=0$, behind the shock. In front of the shock a free vibration exists. What is the nature of the free vibration? Assume that the vibration behind the shock is due to the step pressure increase and that the vibration in front of the shock equals the sum of the pressure increase to $P_{0}$ and a pressure decrease back to $\mathrm{P}=0$. Then the total vibration equals the sum of two step responses, such that

$$
\begin{aligned}
\sigma_{\mathrm{p}}(\mathrm{T}) & =\mathrm{St} \cdot(1-\cos (\omega \cdot \mathrm{T}))-\left(1-\cos \left(\omega \cdot\left(\mathrm{T}-\frac{\mathrm{x}}{\mathrm{V}_{0}}\right)\right)\right) \\
= & \mathrm{St} \cdot\left(\cos \left(\omega \cdot\left(\mathrm{T}-\frac{\mathrm{x}}{\mathrm{V}_{0}}\right)\right)-\cos (\omega \cdot \mathrm{T})\right)
\end{aligned}
$$

However, this assumption does not compensate shock wave speeds, and the arbitrary selection of $\mathrm{T}=0$ also affects the pipe response.

Effects of the Arbitrary Selection of $\mathbf{T}=\mathbf{0}$. The location of $\mathrm{T}$ $=0$ with respect to the shock wave affects the pipe response. As $\mathrm{x} / \mathrm{V}_{0}$ approaches $\mathrm{T}$, the maximum value of Eq. B35 reduces from $2 \cdot \mathrm{St}$ to 0 . Since the boundary condition is arbitrary, the most that can be claimed is that the maximum precursor stress lies within a range of stresses equal to 0 through 2 . St, which is consistent with the variation in maximum strains observed in tests (Leishear [10]). Tests actually showed that the stresses varied between approximately St and $2 \cdot$ St. The maximum stress is therefore described by Eq. B35.

Effects of the Wave Speed. If the precursor vibration is assumed to travel at the wave speed of the shock, the vibration frequency will appear different to a stationary observer. The higher the wave velocity, the higher the frequency appears. A significant assumption is made at this point in the analysis. The critical velocity defined by Eq. A56 is assumed to be the velocity at which the maximum stress occurs. Accordingly, at this velocity the frequencies before and after the shock are nearly equal, and this assumption provides a requisite boundary condition to apply step response equations to the stresses induced by shock waves in the pipe. The frequency after the shock is assumed to equal the frequency for a tube. For an undamped frequency

$$
\begin{aligned}
\omega & =\mathrm{V} \cdot \gamma \\
\omega_{\mathrm{n}} & =\sqrt{\frac{\mathrm{E} \cdot \mathrm{g}}{\rho \cdot \mathrm{R}^{2}}} \quad \text { (Barez) }
\end{aligned}
$$

Note that Eq. B37 differs from Eq. A58 by a factor of $\sqrt{2}$, hence the significance of assuming maximum stresses occur at the critical velocity. The assumption introduces an approximation based on experimental data. Tests performed by Beltman, et. al. [2], measured after shock frequencies equal to the frequencies predicted by assuming that the pipe vibrates at its damped natural frequency, which is

$$
\omega=\sqrt{1-\zeta^{2}} \cdot \sqrt{\frac{E \cdot g}{\rho \cdot R^{2}}}
$$

Assuming the pipe response frequency equals this equation at the critical velocity, the apparent frequency to a stationary observer can be obtained, such that

$$
\begin{gathered}
\sigma_{\mathrm{p}}(\mathrm{T})=\mathrm{St} \cdot\left(\cos \left(\tau \cdot \omega \cdot\left(\mathrm{T}-\frac{\mathrm{x}}{\mathrm{V}_{0}}\right)\right)-\cos (\omega \cdot \mathrm{T})\right) \\
\tau=\frac{\mathrm{V}_{0}}{\mathrm{~V}_{\mathrm{cr}}}
\end{gathered}
$$

Maximum Damped Precursor Stress. To determine the damped maximum precursor stress, Eq. B39 can be rewritten similar to Eq. B32, by recognizing that the exponential function increases with respect to time. Then,

$\sigma_{\mathrm{p}}(\mathrm{T})=-2 \cdot \mathrm{P}_{0} \cdot \frac{\left(\mathrm{OD}^{2}+\mathrm{ID}^{2}\right)}{\left(\mathrm{OD}^{2}-\mathrm{ID}^{2}\right)} \cdot \mathrm{H}\left(\frac{\mathrm{x}}{\mathrm{V}_{0}}-\mathrm{T}\right)$
$\left(1-\frac{\left.\mathrm{e}^{\tau \cdot \zeta \cdot \omega \cdot\left(\mathrm{T}-\frac{\mathrm{x}}{\mathrm{V}_{0}}\right)} \cdot \cos \left(\left(\mathrm{T}-\frac{\mathrm{x}}{\mathrm{V}_{0}}\right) \cdot \tau \cdot \omega \cdot \sqrt{1-\zeta^{2}}\right)-\mathrm{atan}\left(\frac{\zeta}{\sqrt{1-\zeta^{2}}}\right)\right)}{\sqrt{1-\zeta^{2}}}\right)$

\section{After Shock Free Vibration Stresses}

The stresses behind the shock due to free vibrations are assumed to be identical to the precursor vibrations, except that the exponential function decreases with respect to time. That is,

$$
\begin{aligned}
& \sigma_{\mathrm{a}}(\mathrm{T})=-2 \cdot \mathrm{P}_{0} \cdot \frac{\left(\mathrm{OD}^{2}+\mathrm{ID}^{2}\right)}{\left(\mathrm{OD}^{2}-\mathrm{ID}^{2}\right)} \cdot \mathrm{H}\left(\mathrm{T}-\frac{\mathrm{x}}{\mathrm{V}_{0}}\right) \\
& \left(1-\frac{\mathrm{e}^{-\tau \cdot \zeta \cdot \omega \cdot\left(\mathrm{T}-\frac{\mathrm{x}}{\mathrm{V}_{0}}\right)}}{\sqrt{1-\zeta^{2}}} \cdot \cos \left(\left(\mathrm{T}-\frac{\mathrm{x}}{\mathrm{V}_{0}}\right) \cdot \tau \cdot \omega \cdot \sqrt{1-\zeta^{2}}\right)-\mathrm{atan}\left(\frac{\zeta}{\sqrt{1-\zeta^{2}}}\right)\right.
\end{aligned}
$$

Similar to the precursor stress, this stress varies between a maximum predicted by Eq. B42 and zero. Again, a range of stresses are expected along the pipe wall, and Eq. B42 predicts the maximum possible stresses along the pipe. 


\section{Maximum Stresses and Damping}

The maximum stress expressed by Eq. B33, $\sigma(\mathrm{T})=\sigma_{\mathrm{p}}+\sigma_{\mathrm{S}}+\sigma_{\mathrm{a}}$, can be determined once damping is approximated. Damping, $\zeta$, was approximated in the forms of structural, $\zeta_{\mathrm{s}}$, and fluid damping, $\zeta_{\mathrm{f}}$, such that

$$
\zeta=\zeta_{\mathrm{f}}+\zeta_{\mathrm{s}}
$$

Structural Damping Factor. An approximation for structural damping (Lazan [11]) was described in the literature as

$$
\begin{gathered}
\varsigma_{\mathrm{s}}=\frac{\mathrm{Ds} \cdot \mathrm{E}}{2 \cdot \pi \cdot \sigma_{\mathrm{ra}}^{2}} \\
\mathrm{D}=\left(\frac{\sigma_{\mathrm{ra}}}{\sigma_{\mathrm{e}}}\right)^{2.3}+6 \cdot\left(\frac{\sigma_{\mathrm{ra}}}{\sigma_{\mathrm{e}}}\right)^{8} \\
\sigma_{\mathrm{ra}}=2 \cdot \mathrm{St}
\end{gathered}
$$

where $\mathrm{D}$ is the specific damping for a structural material The stress range, $\sigma_{\text {ra }}$, is the magnitude of the completely reversed stress, and the fatigue strength limit is $\sigma_{\mathrm{e}}$.

Fluid Damping Factor. The fluid damping factor was determined by relating the thermodynamic changes in the fluid properties to a dynamic amplification factor. For a step response, a dynamic amplification factor, $i$, is expressed in terms of the percent overshoot (P.O.) as

$$
\mathrm{i}=1+\text { P.O. }=\left(1+\mathrm{e}^{\left.\left[\frac{-\zeta \cdot \pi}{\sqrt{1-\zeta^{2}}}\right]\right)}\right.
$$

Expressing P.O. in terms of thermodynamic properties (Leishear [3]),

$$
\begin{gathered}
\varphi=\frac{\Delta \mathrm{h}-\mathrm{Tamb} \cdot \Delta \mathrm{s}}{\Delta \mathrm{h}} \\
\phi=\mathrm{P} . \mathrm{O} . \\
\zeta_{\mathrm{f}}=\frac{-\ln (\phi)}{\left.\left(\ln (\phi)^{2}+\pi^{2}\right)^{\frac{1}{2}}\right)}
\end{gathered}
$$

where $\Delta \mathrm{h}$ and $\Delta \mathrm{s}$ are the change in enthalpy and entropy respectively, Tamb is the ambient temperature, and $\Phi$ is the ratio of available to theoretical work for a compression process. By substitution, the total damping in the wake of the shock is defined by

$$
\zeta=\frac{-\ln (\phi)}{\left(\left(\ln (\phi)^{2}+\pi^{2}\right)^{\frac{1}{2}}\right)}+\frac{\text { Ds } \cdot \mathrm{E}}{2 \cdot \pi \cdot \sigma_{\mathrm{ra}}^{2}}
$$

In front of the shock, fluid damping is absent and the total damping equals the structural damping only. This affects the frequency in front of the shock, since the damped frequency approximately equals

$$
\sqrt{1-\zeta_{\mathrm{s}}^{2}} \cdot \omega
$$

Consequently, the frequency is higher in front of the shock, than behind the shock. Equation B52, by itself, is inadequate to describe the behavior of the precursor vibration, since observed vibration magnitudes are consistent with the total damping rather than the structural damping.

\section{Maximum Stress When the Critical Velocity is not Considered}

If the maximum stress is assumed to be independent of the critical velocity, the maximum stress can be determined by Eq. B33 and substituting

$$
\tau=1
$$

into the constitutive equations. The net result of this substitution is that the DLF's will be conservatively higher than when the critical velocity was compensated (See Fig. 3). In thick wall solutions, the critical velocity and frequencies are yet to be clearly understood, but this approximation can be used to find a conservative, albeit somewhat high, maximum stress, since the maximum stress will be independent of frequency.

\section{REFERENCES}

[1] Simkins, T., 1987,"Resonance of Flexural Waves in Gun Tubes", Technical report, ARCCB-TR-87008, US Army Armament Research, Development and Engineering Center, Watervliet, N. Y.

[2] Beltman, W. M., Burcsu, E. N., Shepherd, J. E., Zuhal, L., 1999, "The Structural Response of Cylindrical Shells to Internal Shock Loading", Journal of Pressure Vessel Technology, 121, ASME, New York, pp. 315-322.

[3] Leishear, R. A., 2007, "Stresses in a Cylinder Subjected to an Internal Shock", PVT-06-1027, Journal of Pressure Vessel Technology, ASME, New York (in press)

[4] Simkins, T., 1994, "Amplification of Flexural Waves in Gun Tubes", Journal of Sound and Vibration, 172, Academic Press Limited, London, England.

[5] Barez, F. W., Goldsmith, and Sackman, J. L., 1979, (a) "Longitudinal Waves in Liquid Filled Tubes, Theory - I" (b) "Experiments - II", Int. Journal of Mechanical Science, 21, Pergammon Press, Great Britain, pp. 213-236.

[6] Leishear, R. A., 2007, "Dynamic Stresses During Water Hammer, A Finite Element Approach", PVT-06-1029, Journal of Pressure Vessel Technology, ASME, New York (in press).

[7] Timoshenko, S., Woinkowski-Krieger, 199, S, "Theory of Plates and Shells", Mc Graw Hill, New York, pp. 4-5, 465-468

[8] Leishear, R. A. 2006, "Comparison of Theoretical to Experimental Pipe Strains During Water Hammer", PVP2006-ICPVT11-93522, Proceedings PVP2006-ICPVT-11, ASME, New York.

[9] Lubliner, J., 1990,"Plasticity Theory", Macmillan Pub., N.Y., pp. $202-205$.

[10] Thomson, W. T., 1993, "Theory of Vibration with Application"s, Prentice Hall, Inc., New Jersey, pp. 1-192.

[11] Lazan., B. J., 1975, "Structural Damping: Energy Dissipation Mechanisms in Structures, with Particular Reference to Material Damping”, ASME, New York, pp. 1 - 34. 